of tip-induced electric field enhancement, may provide further information on local carrier dynamics. Development of the system so that it can operate in ultra-high vacuum may make it suitable for use with a wider variety of samples.

The microscopy technique demonstrated by Eisele and colleagues is expected to play an important role in advancing nanoscale science and technology. As is discussed above, a variety of time-resolved SPM techniques have already been realized ${ }^{4-8}$. In addition, by combining a spin-polarized tip and pump-probe pulse using a bias voltage, the investigation of inelastic tunnelling has provided information on the spin dynamics in single atoms ${ }^{12}$. Because the nanoscale properties of materials differ from those in bulk structures due to various factors such as the morphology, temperature, band gap, dielectric function and electron-phonon coupling strength, the complementary use of these microscopy techniques is expected to be important for exploring the carrier and spin dynamics in nanoscale structures. In particular, combining microscopy techniques with local excitation using plasmonic superfocusing ${ }^{13}$ may be interesting in the future. The successive development of novel microscopy techniques based on new ideas will greatly aid the further development of nanoscale science and technology.

Hidemi Shigekawa, Shoji Yoshida and Osamu Takeuchi are at the Faculty of Pure and
Applied Sciences, University of Tsukuba, Tsukuba 305-8571, Japan.

e-mail:hidemi@ims.tsukuba.ac.jp

References

1. Eisele, L. et al. Nature Photon. 8, 841-845 (2014).

2. Shah, J. Ultrafast Spectroscopy of Semiconductors and Semiconductor Nanostructures (Springer, 1999).

3. Krausz, F. \& Ivanov, M. Rev. Mod. Phys. 81, 163-234 (2009).

4. Wu, H. J., Nishiyama, Y., Narushima, T., Imura, K. \& Okamoto, H. Appl. Phys. Express 5, 062002 (2012).

5. Wu, S. W. \& Ho, W. Phys. Rev. B. 82, 085444 (2010).

6. Cocker, T. L. et al. Nature Photon. 7, 620-625 (2013).

7. Terada, Y., Yoshida, S., Takeuchi, O. \& Shigekawa, H. Nature Photon. 4, 869-874 (2010).

8. Yoshida, S. et al. Nature Nanotech. 9, 588-593 (2014).

9. Ulbricht, R., Hendry, E., Shan, J., Heinz, T. F. \& Mischa, B. Rev. Mod. Phys. 83, 543-586 (2011).

10. Lucas, M. \& Riedo, E. Rev. Sci. Instrum. 83, 061101 (2012).

11. Zhang, R. et al. Nature 498, 82-86 (2013).

12. Loth, S., Etzkorn, M., Lutz, C. P., Eigler, D. M. \& Heinrich, A. J. Science 329, 1628-1630 (2010).

13. Neacsu, C. C. et al. Nano Lett. 10, 592-596 (2010).
TRANSPARENT ELECTRODES

\section{Fractal future}

Fractals are beautiful patterns based on the principle of self-similarity. Wellknown examples include the branching arrangements of trees, river networks or blood vessels. Now researchers have discovered that fractals can be put to good use in photonics by creating transparent ultrathin metallic electrodes with superior optoelectronic properties.

Transparent electrodes based on conducting oxides such as indium tin oxide or zinc oxide play a crucial role in devices like solar cells, LEDs and displays. However their performance is far from ideal; they are costly, brittle and not well suited to use with some organic materials. As a result, much research is devoted to finding alternatives. One option being explored is the use of ultrathin metal layers. Farzaneh Afshinmanesh and co-workers at the Geballe Laboratory for Advanced Materials in Stanford, USA as well as the Institute of Photonic Sciences and the Catalan Institution for Research and Advanced Studies in Barcelona, Spain have demonstrated that ultrathin aluminium electrodes formed with a fractal geometry have optoelectronic properties superior to their metal counterparts in a grating or grid geometry (Nano Lett. 14, 5068-5074; 2014).

When it comes to transparent electrode design, a high optical transmission is a key figure of merit. The authors studied the performance of aluminium electrodes of various designs that were integrated into
Hilbert fractal

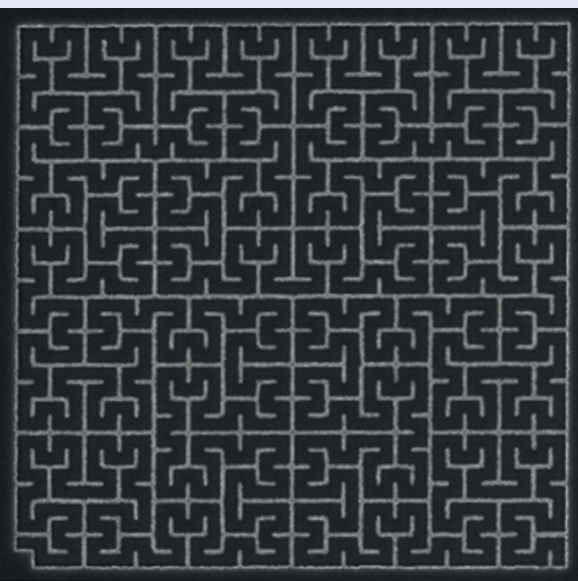

a silicon photodetector. The investigated structures included linear gratings, grids and Hilbert and Peano fractal geometries. The wavelength- and polarization-resolved photocurrent responses of the detector revealed broadband responsivity in the $450-750 \mathrm{~nm}$ range without any polarization dependence apart from the case of the grating. Importantly, detectors featuring the Hilbert and Peano fractal shaped electrodes (pictured) benefited from significantly improved responsivity: they were about 40\% and $34 \%$ better, respectively, compared with the grid, whereas the increase was $7 \%$ and $2 \%$, respectively, compared with the grating.

Breaking down the fractal structures into regions where the metallic wires
Peano fractal

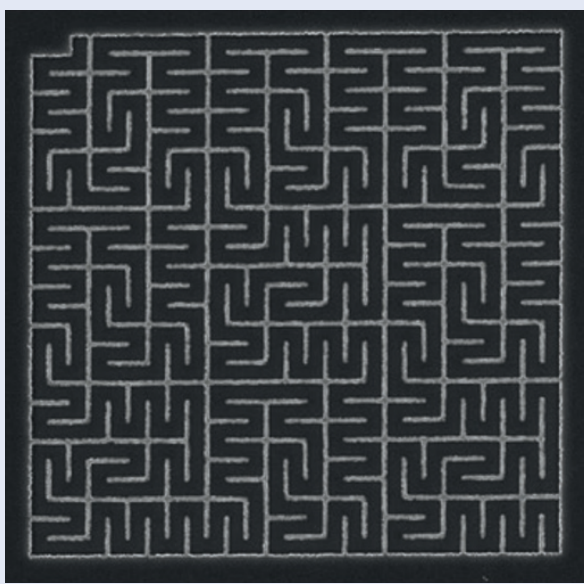

are horizontally and vertically parallel helps elucidate the physics behind the observations. Horizontal gratings transmit primarily vertically polarized light, whereas the opposite is true for vertical gratings. As a result, reflection imaging reveals various bright and dark areas along the surface of the fractal, corresponding to areas of low and high transmission, respectively.

Following further research such fractal electrodes could prove useful in cameras and displays as well as solar cells. In the latter case, the properties of fractal networks may also be helpful for light trapping.

MARIA MARAGKOU 